\title{
Patient Satisfaction Questionnaire for Medical Students' Performance in a Hospital Outpatient Clinic: A Cross-Sectional Study
}

\author{
Marita Fadhilah, ${ }^{1,5}$ Yasutomo Oda, ${ }^{2}$ Sei Emura, ${ }^{3}$ Tsuneaki Yoshioka, ${ }^{1}$ \\ Shunzo Koizumi, ${ }^{1}$ Hirotaka Onishi ${ }^{4}$ and Takanobu Sakemi ${ }^{2}$ \\ ${ }^{1}$ Department of General Medicine, Saga University Hospital, Saga, Japan \\ ${ }^{2}$ Education and Research Center for Comprehensive Community Medicine, Saga Medical School, Saga, Japan \\ ${ }^{3}$ Center for Graduate Medical Education Development and Research, Saga Medical School, Saga, Japan \\ ${ }^{4}$ International Research Center of Medical Education, University of Tokyo, Tokyo, Japan \\ ${ }^{5}$ Faculty of Medicine and Health Sciences, Syarif Hidayatullah Islamic State University, Jakarta, Indonesia
}

\begin{abstract}
Medical education in Japan has undergone significant reforms. Patient perspective and outcome have been highly valued in curricular reforms. Therefore, we evaluated an undergraduate curriculum particularly on communication skills by comparing outpatient satisfaction before and after the reforms implemented at Saga Medical School. Cross-sectional study was conducted at the General Medicine Clinic of Saga University Hospital in 1999 and 2009. A total of 729 newcomer patients evaluated 159 students; namely, 287 patients evaluated sixth-year medical students $(n=82)$ in 1999, and in 2009, 442 patients evaluated fifth-year medical students $(n=77)$. Students interviewed newcomer patients prior to a faculty's clinical examination. After a student-patient encounter, the patient was asked to fill in six-item Patient Satisfaction Questionnaire (PSQ) developed by the American Board of Internal Medicine. Mixed model two-way analysis of variance (ANOVA) with covariant of students' gender was conducted. Effect sizes were calculated to evaluate the amplitude of influence. The average score in 2009 was significantly higher than that in 1999 (3.63 \pm 0.62 versus $3.36 \pm 0.66 ; p<0.001)$. Since the "encouraging and answering questions" and "clear explanations" were lower than those of the other items (3.24 \pm 0.98 and $3.46 \pm 0.85)$, these two items showed the most significant improvements (Phi coefficient $=0.31$ and $0.24, p<0.001$ ). Thus, students' performance has improved since 1999, which may represent the success of curricular reforms at Saga Medical School. We propose that "encouraging and answering questions" and "clear explanations" should be emphasized in interview training.
\end{abstract}

Keywords: communication skills; curricular reforms; educational outcomes; patient satisfaction; students' evaluation Tohoku J. Exp. Med., 2011, 225 (4), 249-254. C 2011 Tohoku University Medical Press

Medical education in Japan has undergone significant reforms both in undergraduate and postgraduate levels in the past two decades. One of the most prominent changes was model core curriculum (MCC) issued by Subcommittee for Research and Development of Medical Education Programs in 2001 in Ministry of Education, Culture, Sports, Science and Technology. MCC outlined essential core components of the undergraduate medical education program in knowledge, skills and attitude domains. Accordingly, effective learning and teaching strategies such as problem-based learning (PBL) and students' participation in patient care for clinical clerkship were emphasized (Onishi and Yoshida 2004; Kozu 2006).
Related to MCC, implementation of Common Achievement Test (CAT) has given a strong impact on undergraduate medical education, especially with regards the preclinical curriculum. CAT is aimed at quality assurance of medical students before allowing them to proceed to clinical clerkship. CAT consists of two parts, 300 items of multiple-choice questions in Computer-based Testing (CBT) for knowledge and six- to eight-station Objective Structured Clinical Examination (OSCE) for clinical skills and attitude (Onishi and Yoshida 2004; Kozu 2006; Suzuki et al. 2008).

In response to MCC and CAT, many medical schools reformed their curriculum. A survey in 2009 indicated that

Received August 15, 2011; revision accepted for publication October 26, 2011. doi: 10.1620/tjem.225.249

Correspondence: Marita Fadhilah, M.D., Department of General Medicine, Saga University Hospital, 5-1-1 Nabeshima, Saga Shi, Saga 849-8501, Japan.

Faculty of Medicine and Health Sciences, Syarif Hidayatullah Islamic State University, Jl. Ir. H. Juanda no. 95, Ciputat 15419, Jakarta, Indonesia.

e-mail: maritafadhilah@uinjkt.ac.id 
73 out of 80 Japanese medical schools $(91.3 \%)$ have adopted MCC and all medical schools have joined CAT system. PBL was implemented in 70 medical schools, while most (78 schools) introduced clinical clerkship program in which medical students participate more actively in patient care rather than observational learning. Recently, all medical schools implemented the Introduction to Clinical Medicine (ICM) program, which covers medical interview skills, physical examination skills, evidence based medicine, etc. before clinical clerkship. Sixty-three medical schools have already established well-equipped clinical skill laboratories that help support the ICM program. Forty-seven medical schools have also incorporated Simulated Patients (SP) in their skill training prior to clinical clerkship (Association of Japanese Medical College 2009a, 2009b). Yet, Japanese medical education scene lacks an evidence of curricular reform after clinical clerkship was implemented.

Meanwhile, evaluating these curricular reforms is also essential for continuous educational development. Donald Kirkpatrick developed a framework for evaluating training programs (Kirkpatrick 1967). Many evaluation surveys merely address students' reactions (lowest level) or acquisition of knowledge, skills and attitudes (second lowest level) of Kirkpatrick's four levels. No evaluation study for curricular reforms in Japan has tried to measure the third and fourth levels of Kirkpatrick's by measuring whether students are able to achieve the learning outcomes in actual clinical settings and to make impacts on patients.

Indeed, curricular reforms must serve patient care in the end. Therefore, patient satisfaction and outcome should be highly valued when assessing curriculum development. The objective of this study is to evaluate the undergraduate curriculum, especially students' communication skills, by comparing outpatient satisfaction before and after Japanese medical education reforms implemented at Saga Medical School (SMS).

\section{Educational context at SMS}

In the first decade of this century, six-year SMS curriculum for undergraduate students was reformed drastically from teacher-centred and discipline-based to student-centred and integrated. The "Introduction to Health Care" program conducted in the first two years of preclinical education, which guides how students learn to practice health care, was expanded from 30 to 120 hours. It includes 10 days of early clinical attachment and 12 hours of clinical skill training including communication training with SP.

Basic medical sciences for second-year students was integrated and incorporated with organ-based clinical medicine. Fifty-five weeks of PBL was implemented as the main teaching strategy for clinical medicine throughout the third and fourth years (Oda and Koizumi 2008). In the PBL program, the total hour of didactic lecture in the large classroom was reduced to less than $45 \%$ of the old curriculum (from 1,177 to 527 hours). Instead, small group discussions using case scenarios under facilitation of a tutor (from 5 to 273 hours) and self-directed learning (from 134 to 757 hours) were allotted (Oda and Koizumi 2008).

CAT (CBT and OSCE) was introduced to SMS at the end of pre-clinical curriculum (fourth-year) to assure students' clinical skills mastery before entering the clinical clerkship program since 2006. Related to OSCE, the ICM program in the third and fourth years was expanded from 60 to 124 hours. In addition, as the formative assessment of clinical clerkship in fifth and sixth years, advanced-level OSCE was implemented as part of the graduation examination.

To enrich basic clinical skill training, a skill laboratory was established and full-time clinical skill trainers were employed. Moreover, SMS has organized SP group since 2003. A total of 38 hours of communication training sessions for medical students in pre-clerkship were held and 314 SPs were involved in 2010.

\section{Methods}

Setting and design

Cross-sectional study was conducted in General Medicine Clinic (GMC) of Saga University Hospital in 1999 and 2009. This study was approved by Ethics Committee of Saga University Hospital.

\section{Outcomes measures}

As a work-based assessment tool for physicians' performance assessment in their daily practice (Norcini 2003), we selected Patient Satisfaction Questionnaire (PSQ) developed by American Board of Internal Medicine (ABIM). PSQ is a validated instrument that makes physicians' humanistic quality assessable from the viewpoint of actual patients (PSQ Project Co-Investigators 1989).

According to the previous study (Oda et al. 2004), we selected six-items from original ten-items ABIM-PSQ with five-point Likert scale (1-poor, 2-fair, 3-good, 4-very good and 5-excellent) as described in Table 2. It because to adapt them to the undergraduate curriculum in SMS. Also developers of ABIM-PSQ reported that any items more than four out of ten would not deteriorate the reliability of score (Yonke and Foley 1991). In this study, alpha coefficient of sixitems was 0.83 .

Three investigators independently translated ABIM-PSQ into Japanese and later discussed the discrepancy to adjust. The contents of back-translation into English were authorized by ABIM. The official Japanese translated version of PSQ has been approved and given permission by ABIM (Oda et al. 2004).

\section{Participants}

A two-week clinical clerkship at the GMC has been mandatory for all of medical students in clinical clerkship. In 1999, we obtained PSQ scores from 287 new patients, who had been interviewed by 82 final (sixth)-year medical students. In 2009, we obtained PSQ scores from 442 newcomer patients, who had been interviewed by 77 fifthyear medical students. Totally we used PSQ scores from 729 newcomer patients interviewed by 159 medical students. Both students' groups have passed the same selection process, admission policy and entrance examination. We excluded patients with cognitive impairment or severe symptom that prevented them from filling in the PSQ. 


\section{Data gathering process}

Within two weeks of clinical clerkship program in GMC, students independently interviewed several newcomer patients without faculty supervision. In addition, students have no access to the PSQ form and receive no feedback of their own PSQ scores. Just after the initial student-patient encounter, a research assistant explained the objectives of this research to patients, provided the PSQ form, and asked patients to anonymously fill in the form. Completion time was flexible and patients were ensured privacy while completing the PSQ. We guaranteed those patients that the result would not affect their treatment and/or management. Completed questionnaires were collected by the research assistant. After all the processes above were finished, preceptors interviewed and examined those patients.

\section{Statistical analysis}

Firstly, we classified poor and fair in PSQ as low performance and good, very good and excellent as high performance. Then we performed chi-square test for each PSQ item between two timings of 1999 to 2009. Phi coefficients were also calculated for effect sizes and classified into three levels of improvement, small, medium and large. The score of Phi coefficient larger than 0.3 was considered as a medium to large improvement (Mizumoto and Takeuchi 2011).

As the further analysis, the two factors, two timings of years (1999 and 2009; between subject factor) and six PSQ items (within subject factor), were incorporated into mixed model two-way analysis of variance (ANOVA) after converting the PSQ scores into continuous scale. To remove the influence of students' gender as confounding factor, this factor was also incorporated into the analysis as a covariant. For the test of within-subjects effects involving more than one degree of freedom, Huynh-Feldt epsilon correction was used. For a pair-wise comparison, "Least Significant Difference" was adopted.

We measured effect sizes (eta squared) for the factors of timing and PSQ item (Kline 2004). Partial eta squared output from SPSS was converted into eta squared with a standard formula (Hori 2011). The scores of eta squared were estimated into three levels of improvement, small, medium and large. The score of eta squared more than 0.06 was considered as a medium to large improvement.

Statistical analysis was performed with SPSS 16.0. $P$ value of less than 0.05 was considered significant.

\section{Results}

Characteristics of medical student and patient demographics (Table 1) did not show any significant difference between 1999 and 2009.

Table 2 shows the percentages of PSQ item responses both in 1999 and 2009. In 2009, students' percentages rated as low performance in PSQ items were decreased, especially for "encouraging and answering questions" (Q5) and "clear explanation" (Q6).

Percentages of students rated as low performance were compared between 1999 and 2009 by chi-square test (Table 3). Q5 "encouraging and answering questions" gained medium to large improvement, 0.31 of Phi coefficient and Q6 "clear explanations" gained small to medium improvement, 0.24 of Phi coefficient.

After converting the data into continuous scale, overall average of PSQ scores was $3.52 \pm 0.65$. Average score in 2009 was significantly higher than that in $1999(3.63 \pm 0.62$ versus $3.36 \pm 0.66 ; \mathrm{F}_{(1,720)}=30.55, p<0.001$; Table 4$)$ and 0.041 of eta squared revealed small to medium improvement. PSQ scores indicated significant differences among six items $\left(\mathrm{F}_{(3.8,3600)}=9.76, p<0.001\right)$ and 0.013 of eta

Table 1. Characteristics of students and patients.

\begin{tabular}{cll}
\hline & \multicolumn{1}{c}{1999} & \multicolumn{1}{c}{2009} \\
\hline Medical student & 82 & 77 \\
- Male (\%) & $47(57.3)$ & $42(54.5)$ \\
- Female (\%) & $35(42.7)$ & $35(45.5)$ \\
Patient & 287 & 442 \\
- Male (\%) & $136(47.4)$ & $212(48)$ \\
- Female (\%) & $151(52.6)$ & $230(52)$ \\
- Age (range) & $50.3(16-88)$ & $56.9(15-91)$ \\
PSQ rating & 287 & 442 \\
- For male student (\%) & $155(54)$ & $232(52.5)$ \\
- For female student (\%) & $132(46)$ & $210(47.5)$ \\
\hline
\end{tabular}

Table 2. Percentages of PSQ scores in 1999 and 2009.

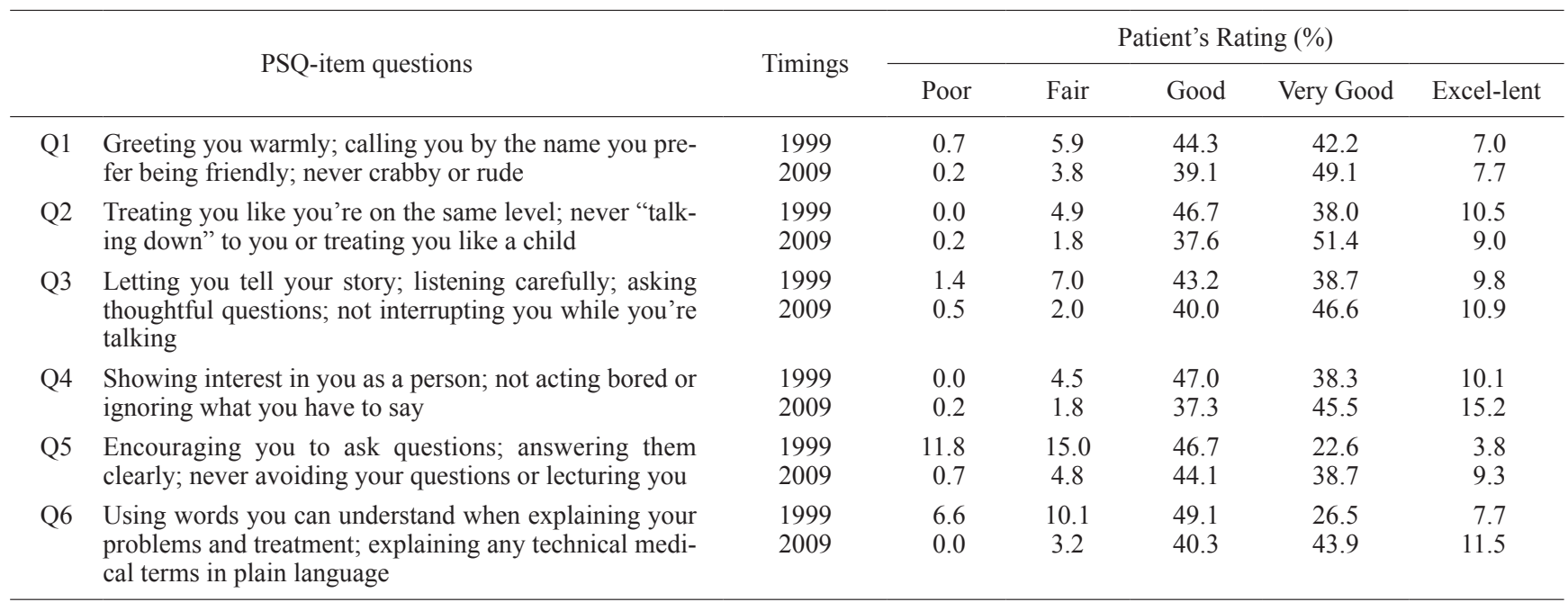


Table 3. Percentages of low performance students by PSQ and its effect sizes.

\begin{tabular}{llcccc}
\hline \multicolumn{1}{c}{ PSQ Items } & $\begin{array}{c}1999 \\
(\%)\end{array}$ & $\begin{array}{c}2009 \\
(\%)\end{array}$ & $\begin{array}{c}\text { Chi-square } \\
(p)\end{array}$ & Phi coefficient \\
\hline Q1 & Greeting and friendliness & 6.6 & 4.0 & 0.123 & 0.06 \\
Q2 & Respect for patients & 4.9 & 2.0 & 0.031 & 0.08 \\
Q3 & Careful listener & 8.4 & 2.5 & $0.001>$ & 0.14 \\
Q4 & Showing interest personally & 4.5 & 2.0 & 0.053 & 0.07 \\
Q5 & Encouraging and answering questions & 26.8 & 5.5 & $0.001>$ & 0.31 \\
Q6 & Clear explanations & 16.7 & 3.2 & $0.001>$ & 0.24 \\
\hline
\end{tabular}

Table 4. PSQ items and mean (SD) of item scores.

\begin{tabular}{llcc}
\hline \multicolumn{1}{c}{ PSQ Items } & $\begin{array}{c}1999 \\
(n=287)\end{array}$ & $\begin{array}{c}2009 \\
(n=442)\end{array}$ \\
\hline Q1 & Greeting and friendliness & $3.49(0.74)$ & $3.60(0.70)$ \\
Q2 & Respect for patients & $3.54(0.75)$ & $3.67(0.67)$ \\
Q3 & Careful listener & $3.48(0.82)$ & $3.65(0.72)$ \\
Q4 & Showing interest personally & $3.54(0.74)$ & $3.74(0.74)$ \\
Q5 & Encouraging and answering questions & $2.92(1.00)$ & $3.45(0.90)$ \\
Q6 & Clear explanations & $3.18(0.95)$ & $3.65(0.73)$ \\
\hline \multicolumn{2}{c}{$\begin{array}{l}\text { Average } \\
\text { (overall average }=3.52(0.65))\end{array}$} & $3.36(0.66)$ & $3.63(0.62)$ \\
\hline
\end{tabular}

squared revealed small difference. Pair-wise comparisons among PSQ six-item scores indicated that "encouraging and answering questions" (Q5) item score was significantly lower than any other items $(3.24 \pm 0.98)$, and that "clear explanations" (Q6) item score was lower than other four items $(3.46 \pm 0.85)$.

Furthermore, the interaction between factors of PSQ and year was also significant $\left(\mathrm{F}_{(3.8,3600)}=25.03, p<0.001\right)$ and eta squared revealed small to medium difference (eta squared $=0.034$ ). But interaction between factors of PSQ and students' gender was no significant difference $\left(\mathrm{F}_{(3.8,3600)}\right.$ $=0.82, p=0.50)$. And also, female students' scores were not significantly different than male students' $\left(\mathrm{F}_{(1,720)}=0.15\right.$, $p=0.70)$.

\section{Discussion}

The results showed that patient satisfaction was improved between 1999 and 2009, especially with regards to the "encouraging and answering questions" and "clear explanations" items. However, the conclusion of this crosssectional study should be drawn cautiously. Firstly, threats to internal validity should be considered, such as history. Other types of threats to internal validity, such as maturation, selection, or subject mortality are not applicable to this situation, because the clinical clerkship program in GMC was only one time for each student. Threat of history means much for this study because patients' perception of relationship with physicians becomes much stricter in these 10 years. Nevertheless average patient satisfaction score for the fifth-year medical students in 2009 was significantly higher than that for the sixth-year students in 1999. The most probable reason for this improvement is the successful shift from teacher-centered to student-centered learning to motivate students' self-directed learning rather than passing learning.

Due to rotation of clinical clerkship, we involved different grades of students, sixth-year students in 1999 and fifth-year students in 2009. The findings showed that the fifth-year students' performance as rated by patients was higher than that of the sixth-year students. Since both fifthyear and sixth-year students are in the same stage of clinical clerkship, those results referred to successfully curricular reforms at SMS rather than quality of students themselves. The other reason is the sixth-year students in 1999 had fewer opportunities to interview patients (each student interviewed 3.5 patients in average) than the fifth-year students in 2009 who interviewed 5.7 patients in average for each within two-week rotation. Experienced with more actual patients really have strong impact on students.

Of particular importance in this study were the low scores in the "encouraging and answering questions" and "clear explanations" items, which showed significant improvement in 2009. Several reasons for low scores in 1999 in these items can be discussed. Firstly, the results might come from weaknesses in our ICM program, meaning that the pre-clerkship curriculum did not sufficiently emphasize education and motivation of patients (Oda et al. 2004). Secondly, other items in medical interview, such as the "careful listener" and "respect for patients," are relatively passive. It is more difficult to 
practice "encouraging and answering questions" and "clear explanations" in undergraduate curricula (Oda et al. 2004). Thirdly, these 'process' skills (the way we communicate) are more complicated task to acquire than 'content' skills (what we communicate). Kurtz et al. (2003) highlighted that "facilitative response" and "clarification", which is similar to the "encouraging and answering questions" and "clear explanations" items, refers to "process' rather than 'content' skills. Moreover, if we explore the PSQ items in more detail, these two low-scored items also appear to be more strongly influenced by the mastery of clinical knowledge not just communication skills.

The average scores of "encouraging and answering questions" and "clear explanations" in 2009 showed the highest improvement. Several steps might be effective for this improvement. The first step was the implementation of the ICM program and the resulting of standardized communication training encouraged students to have more active role in medical interview. Other steps, such as CAT including the OSCE program, PBL allowing them to apply basic science to actual patients, and CBT assuring certain level of knowledge seem to play important roles.

The "Introduction to Health Care" program has allowed students early clinical exposure to medical practice and basic clinical skills training for a longer duration during their first and second years. In addition, the standardized communication training has helped enable discrimination between low and high performance students, as well as enforcing students to learn in a uniform manner. As a result, students are in a position whereby they can learn and gain confidence in the areas they need to explore further. These factors might be useful in helping low performance students at an early stage.

This study has three strengths. Firstly, it is the first quantitative study, in which outpatients rated the improvement in students' performance, after introduction of the recent reforms in Japanese undergraduate education. Secondly, we used the PSQ, which seemed to successfully reflect patients' true feelings after their experience with the students. Sometimes patients are unable to express their feelings coherently. Therefore, the PSQ proved to be a valuable tool. Thirdly, based on the direct observations and patients' free comments that were written in below PSQ form, the majority of patients welcomed medical student involvement during the clinical encounters. Many medical educators hesitate to take medical students to real patients to avoid any risk of patient safety. In our educational practice, it was a good opportunity for hands-on clinical experience, especially since during clinical clerkship students mainly observe rather than actively participate in clinical care (Teo 2007).

Despite its findings, this study has limitations. Firstly, within a decade there are many factors involved in improvement of students' performance, above mentioned as a threat to internal validity. Not only the success of new SMS curriculum, but also introduction of OSCE, ICM program and development of skills laboratory. Other confounding factor also involved, such as the patients themselves, the hospital facilities, the change of health care situation etc. Secondly, both student groups had only a few opportunities to interview patients within the two-week rotation. With more practice, therefore, both communication skills and patient rating scores might have shown further improvement.

PSQ provided valuable evidence of the importance of student evaluation from the viewpoint of the patient. In general, patient ratings tend to provide a global impression rather than objective evaluation. Since they are expert in their life worlds, patients' outcomes are the best measures of the quality of doctors for the public, the patients and doctors themselves (Norcini 2003). In the future, assessments by peers, nurses, and faculty members as well as direct observation-based assessment are needed to further clarify the findings of this study.

\section{Conclusions}

In summary, the present study with PSQ scores has highlighted that students' communication skills have improved within the past 10 years, partly meaning the success of SMS curricular reforms and possibly Japanese medical education reforms. In addition, this study also indicates the need for adequate instruction to be focused on "encouraging and answering questions" and providing "clear explanations" in both interview training and the pre-clerkship and clerkship curricula.

\section{Acknowledgments}

This work was supported by Grant-in-Aid for Scientific Research (C) \#21590757 of Japan Society for the Promotion of Science.

\section{Conflict of Interest}

The authors declare that they have no competing interest.

\section{References}

Association of Japanese Medical Colleges. (2009a) Current curriculum in Japanese Medical Education 2009: Explanation of Survey's Results, Tokyo, pp. 1-19 (in Japanese).

Association of Japanese Medical Colleges. (2009b) A White Paper on the Medical School of Japan, Tokyo, pp. 192-271 (in Japanese).

Hori, K. http://www.ec.kagawa-u.ac.jp/ hori/spss/tokidoki24. html\#242 (in Japanese). [Accessed: February 2011]

Kirkpatrick, D.L. (1967) Evaluation of training. In Training and Development Handbook, edited by R. Craig and I. Mittel. McGraw Hill, New York, pp. 87-112.

Kline, R.B. (2004) Beyond Significance Testing: Reforming Data Analysis Methods in Behavioral Research, American Phychological Association, Washington, DC.

Kozu, T. (2006) Medical Education in Japan. Acad. Med., 81, 1069-1075.

Kurtz, S., Silvermann, J., Benson, J. \& Draper, J. (2003) Marrying Content and Process in Clinical Method Teaching: Enhancing the Calgary-Cambridges Guides. Acad. Med., 78, 802-809.

Mizumoto, A. \& Takeuchi, O. http://www.mizumot.com/files/ EffectSize_KELES31.pdf (In Japanese). [Accessed: February 


\section{1]}

Norcini, J.J. (2003) ABC of learning and teaching in medicine: Work based assessment. BMJ, 326, 753-755.

Oda, Y. \& Koizumi, S. (2008) Status of medical education reform at Saga Medical School 5 years after introducing PBL. Kaohsiung. J. Med. Sci., 24 (Suppl 3), S46-53.

Oda, Y., Onishi, H., Yamashiro, S. \& Koizumi, S. (2004) The assessment of undergraduate curriculum of communication skills evaluated by performance measurement using actual outpatient satisfaction. Med. Edu., 35(2), 89-94 (In Japanese).

Onishi, H. \& Yoshida, I. (2004) Rapid change in Japanese medical education. Med. Teach., 26, 403-408.
PSQ Project Co-Investigators. (1989) Final Report on the Patient Satisfaction Questionnaire Project, American Board of Internal Medicine, Philadelphia, PA.

Suzuki, Y., Gibbs, T. \& Fujisaki, K. (2008) Medical education in Japan: a challenge to the healthcare system. Med. Teach., 30, 846-850.

Teo, A. (2007) The current state of medical education in Japan: a system under reform. Med. Educ., 41, 302-308.

Yonke, A.M. \& Foley, R.P. (1991) Overview of recent literature on undergraduate ambulatory care education and a framework for future planning. Acad. Med., 66, 750-755. 